Meta

Journal des traducteurs

Translators' Journal

\title{
Tropes et termes : le vocabulaire de la dégustation du vin
}

\section{Martine Coutier}

Volume 39, numéro 4, décembre 1994

Hommage à Bernard Quemada : termes et textes

URI : https://id.erudit.org/iderudit/002423ar

DOI : https://doi.org/10.7202/002423ar

Aller au sommaire du numéro

Éditeur(s)

Les Presses de l'Université de Montréal

ISSN

0026-0452 (imprimé)

1492-1421 (numérique)

Découvrir la revue

Citer cet article

Coutier, M. (1994). Tropes et termes : le vocabulaire de la dégustation du vin. Meta, 39(4), 662-675. https://doi.org/10.7202/002423ar

\section{Résumé de l'article}

Depuis les années cinquante, l'engouement pour le vin, boisson à forte charge symbolique, est devenu un phénomène culturel qui s'inscrit dans l'évolution des comportements sociaux et alimentaires. La nouvelle approche du vin implique un nouveau public de dégustateurs, dont le profil se situe entre les spécialistes professionnels (œnologues, experts-dégustateurs), et les simples amateurs. Les impressions gustatives ne correspondant pas à une réalité référentielle objectivable et étant soumises à une forte subjectivité, le vocabulaire qui les décrit est marqué par le recours à l'analogie, la métaphore, caractéristique lexicale renforcée par la composante hédoniste de la dégustation. Cet article se propose d'analyser les tropes lexicaux relevés dans des comptes rendus de dégustation, à travers deux champs thématiques sources de métaphores : le champ du corps humain et celui de la réalité spatiale. La mise en évidence des rapports et des réseaux analogiques et thématiques ainsi que leur ordonnancement permet de dessiner, derrière ce qui peut paraître une imagination lyrique et débridée, un ensemble construit autour de références communes et tendant vers une certaine cohérence.
Ce document est protégé par la loi sur le droit d'auteur. L'utilisation des services d'Érudit (y compris la reproduction) est assujettie à sa politique d'utilisation que vous pouvez consulter en ligne.

https://apropos.erudit.org/fr/usagers/politique-dutilisation/ 


\title{
TROPES ET TERMES : LE VOCABULAIRE DE LA DÉGUSTATION DU VIN
}

\author{
MARTINE COUTIER \\ CNRS-INaLF, Besançon, France
}

\begin{abstract}
Résumé
Depuis les années cinquante, l'engouement pour le vin, boisson à forte charge symbolique, est devenu un phénomène culturel qui s'inscrit dans l'évolution des comportements sociaux et alimentaires. La nouvelle approche du vin implique un nouveau public de dégustateurs, dont le profil se situe entre les spécialistes professionnels (conologues, expertsdégustateurs), et les simples amateurs. Les impressions gustatives ne correspondant pas à une réalité référentielle objectivable et étant soumises à une forte subjectivité, le vocabulaire qui les décrit est marqué par le recours à l'analogie, la métaphore, caractéristique lexicale renforcée par la composante hédoniste de la dégustation.

Cet article se propose d'analyser les tropes lexicaux relevés dans des comptes rendus de dégustation, à travers deux champs thématiques sources de métaphores: le champ du corps humain et celui de la réalité spatiale. La mise en évidence des rapports et des réseaux analogiques et thématiques ainsi que leur ordonnancement permet de dessiner, derrière ce qui peut parâtre une imagination lyrique et débridée, un ensemble construit autour de références communes et tendant vers une certaine cohérence.
\end{abstract}

\begin{abstract}
Since the fifties, the great interest in wine, a beverage with highly symbolic implications, has become a cultural phenomenon which is a part of current social and dietary behaviors. A new approach to wine implies a more diversified wine drinking and tasting public whose profile can be located between the specialized professionals (oenologist, vint ner, taster) and the amateur wine lover and drinker. Gustatory impressions do not correspond to an objective referential reality and as these impressions are often highly subjective, the vocabulary describing them is marked by analogy and metaphor, a lexical characteristic which is reinforced by the hedonistic component of wine tasting.

The aim of this paper is to analyse the lexical tropes taken from wine tasting reports, using two thematic fields which have given rise to metaphoric usage, i.e. the theme of the human body and that of spatial relations. A presentation of the thematic and analogical networks as well as their graduated relationships provides a basic set of terms, which at first glance could appear to be the result of a spirited, exuberant and lyrical imagination, but which has been built up around common references and tends to form a coherent whole.
\end{abstract}

Comme tout domaine d'expérience, la dégustation du vin possède un vocabulaire spécifique élaboré et développé par les praticiens de cette activité particulière. Cette activité se distingue cependant de la plupart des autres par deux aspects qui la caractérisent: la nature de son référent, de l'ordre du sensoriel, qui échappe en grande partie à la possibilité d'être objectivé, et le fait qu'elle n'a de sens que par le discours qu'elle engendre: "Aucune substance consommable n'a la même complicité que le vin avec la parole [...] il est le seul produit dont la consommation exige un commentaire, puisque savoir le boire revient à savoir en parler.» (Châtelain-Courtois $1984: 5$ ).

À l'écoute ou à la lecture d'un commentaire de dégustation, le profane ou «buveur de vin moyen» se trouve aussi désemparé ou perplexe que devant un texte relevant d'une discipline dont l'objet lui serait inconnu. Et pourtant, si sa compréhension peut être 
arrêtée par un certain nombre de termes techniques relatifs à la viticulture, à la vinification et à l'analyse sensorielle (botrytisé, extrait sec, macération pelliculaire, rétroolfaction, etc.) exigeant à l'évidence des connaissances et un apprentissage préalables, ce sont souvent les mots en apparence les plus communs qui lui font prendre conscience du caractère particulier, spécifique, «initiatique» du discours de la dégustation et de ce qu'il recouvre:

«Une jolie chair en bouche, de bons tannins, une très belle expression aromatique et une bonne persistance. L'équilibre entre structure et charnu est bien réussi, la matière est belle, l'extraction a été particulièrement soignée. C'est un vin [volnay clos d'Audignac 1989] riche, gras, chaud, qui séduit sans conteste par son caractère athlétique et complexe, son boisé distingué.» (La Revue du vin de France, Suppl. au n 370, déc. 1992: 37).

Il existe certes, dans le vocabulaire du goût, des termes propres, dénotatifs, non ambigus, correspondant à des perceptions sensorielles identifiées et répertoriées : acide, aigre, empyreumatique (= goût de brûlé), sucré, etc. Mais ils sont bien peu nombreux en regard de la réalité à exprimer. Aussi l'indigence de notre langue comme la difficulté inhérente à l'appréhension des perceptions sensorielles expliquent-elles le recours à l'image, à l'analogie, puisant leurs sources dans la langue commune à travers des thèmes de prédilection. Ce qui fait la spécificité et la richesse du vocabulaire qui nous intéresse relève avant tout de la métasémie.

Pour le linguiste, la question est alors de savoir dans quelle mesure les unités d'un discours métaphorique comme celui de la dégustation du vin peuvent être considérées comme des termes. Peut-on évaluer la part de la fonction cognitive et celle de la fonction poétique assurée par ces vocables ? Y a-t-il une cohérence quelconque derrière l'apparent désordre du foisonnement lexical utilisé pour décrire le vin?

L'étude qui suit tente d'apporter des éléments de réponse à ces questions; elle s'appuie sur des relevés qui ont été effectués à partir des comptes rendus de dégustation consignés dans deux revues viniques : La Revue du Vin de France et Le Rouge et le Blanc (voir Bibliographie) entre 1980 et 1993. Elle n'envisage que les unités métaphoriques, adjectifs, noms et verbes, appliqués à la description et l'appréciation olfacto-gustative du vin.

\section{LA DÉGUSTATION DU VIN ET SON VOCABULAIRE}

Telle qu'elle est définie par le Conseil international de la langue française, la dégustation est :

«une opération consistant à expérimenter, analyser et apprécier les caractères organoleptiques et plus particulièrement les caractères olfacto-gustatifs d'un produit. Elle peut être plus ou moins analytique et détaillée, c'est-à-dire tendre à décomposer ses caractères en éléments simples, à rattacher tel ou tel goût à telle substance ou ensemble de substances de référence (dégustation analytique). Elle peut être au contraire plus ou moins globale, c'est$\grave{a}$-dire tendre à exprimer le plaisir ou le déplaisir ressenti (dégustation hédonique).» (Peynaud $1980: 2-3$ ).

On relèvera les verbes analyser, apprécier et exprimer dans cette définition, qui rendent compte du lien indissociable entre dégustation et expression, c'est-à-dire «mise en mots», verbalisation.

Dans l'analyse sensorielle mise en œuvre dans la dégustation du vin, seules les perceptions visuelles disposent de références objectives, mesurables et codifiables, qui n'excluent pas pour autant la variété et la subjectivité dues aux différences individuelles. Il n'en est pas de même pour les perceptions olfactives et gustatives. 
Les nombreuses recherches menées actuellement sur le goût par des spécialistes de disciplines diverses - neurophysiologistes, neurobiologistes, psychophysiologistes, agronomes, sociologues, etc., mettent l'accent sur l'obstacle représenté par les lacunes du vocabulaire gustatif: «le manque de descripteurs (qui) résulte des différences de perception interindividuelles» (Faurion 1992: 22); «le vocabulaire pour parler de ce deuxième sens chimique (le goût) est très au-dessous de la réalité (McLeod 1987: 78); «soumis aux jeux du symbolisme, (le vocabulaire de la dégustation) mêle inextricablement évaluations objectives et valorisations» (Albert 1989: 123).

Les perceptions olfactives et gustatives sont par nature extraordinairement complexes, fugitives et subjectives, et reposent essentiellement sur la mémorisation des odeurs et des saveurs. Leur description peut consister à établir des comparaisons avec le goût d'un aliment ou d'une chose dont on a gardé le souvenir (cela «rappelle» la framboise, la vanille, le miel, le bois, le cuir ...), et être à la base de créations néologiques: cassissé, fruit compoté, figué, muscaté, noiseté, réglissé, tabacé, etc. Elle comporte également une série d'impressions diverses (sensations de volume, de forme, de matière, d'intensité, de durée, sensations tactiles, thermiques, sensations de plaisir), sources d'une expression métaphorique riche et variée.

\section{ÉVOLUTION DU VOCABULAIRE DE LA DÉGUSTATION}

Comme le vin, l'art de déguster est fort ancien. Aussi un certain nombre de termes sont-ils attestés dès le Moyen âge (bon, bonté, douceur au sens de «souplesse», force, franc, puissant etc.) (Henry 1991). Il n'est pas dans notre propos de présenter une histoire de ce vocabulaire, pour laquelle on ne dispose d'ailleurs pas encore de données suffisantes 1 . Mais il est nécessaire d'en tracer les grandes lignes et de donner quelques indications qui permettront d'apprécier son état actuel.

Selon Peynaud (1980: 155), le «langage des goûteurs» (ou «essayeurs», c'est-à-dire ceux qui exerçaient la profession de courtiers-gourmets) des $X^{e}$ et $X V I^{e}$ siècles était limité à un petit nombre de termes de base (non évalué) qui a peu évolué jusqu'au XVIII $\mathrm{s}$. C'est seulement à cette période que le vocabulaire s'est développé et diversifié. À cela, deux raisons: les progrès dans la viticulture et l'élaboration du vin (dégustateur, au sens professionnel du terme, apparaît en 1793), qui favorisent une évolution de la qualité, et l'exigence même de cette qualité, liées aux contraintes commerciales. Il semble que le mouvement se soit dessiné à Bordeaux (Pijassou 1977: 169), où les courtiers se réunissaient pour goûter les vins destinés à être acheminés en Angleterre et dans les autres pays du nord. Et c'est seulement en 1822, dans la Topographie de tous les vignobles connus de A. Jullien, que sont rassemblés pour la première fois dans un «Vocabulaire des termes employés dans cet ouvrage pour désigner les différentes qualités des Vins», près de 90 termes techniques, dont plus de 60 se rapportent à la description des caractères du vin.

Le XIX ${ }^{e}$ siècle est marqué par divers événements politico-économiques qui jalonnent l'histoire de la viticulture (par exemple l'arrachage des vignes et le démantèlement de nombreuses propriétés viticoles pendant la Révolution), mais aussi par les recherches sur le vin ${ }^{2}$ et enfin, à la fin du siècle, par l'épidémie de phylloxéra qui ruine le vignoble français. La reconstruction de celui-ci se fait lentement et partiellement au début du XX ${ }^{\mathrm{e}}$ siècle, et c'est la période suivant la deuxième guerre mondiale qui va marquer une étape décisive pour un essor dans tous les domaines du vin : d'une part les progrès techniques et scientifiques (le diplôme d'œnologue est créé en 1955), d'autre part la concurrence commerciale due au développement des vignobles du «nouveau monde», contribuent à l'amélioration continue de la qualité; l'élévation du niveau de vie et l'évolution des goûts et des comportements alimentaires sont des facteurs déterminants qui favorisent la consommation de vin en l'inscrivant dans un environnement à la fois socio-économique et 
culturel: le vin est devenu un phénomène de société de la fin du $\mathrm{XX}^{\mathrm{e}}$ siècle, un objet d'étude pour les sociologues (Albert 1989; Fischler 1992).

\section{Codification et diffusion du vocabulaire de la dégustation}

L'incidence de cette évolution sur le vocabulaire, dans son élaboration et sa diffusion, ne se fait pas attendre: d'une part les nouveaux professionnels du vin, c'est-à-dire les œnologues et les dégustateurs-experts, tentent de définir et codifier une terminologie indispensable à leur profession, parallèlement aux recherches menées sur la dégustation des produits alimentaires en général (Le Magnen 1962): l'ouvrage technique de Vedel et al., L'Essai sur la dégustation des vins (1972), publié par l'Institut National des Appellations d'Origine, rassemble environ 470 termes relatifs aux caractères gustatifs sur les 900 entrées du «Vocabulaire».

D'autre part, cette terminologie va sortir des laboratoires pour être livrée au public grandissant d'œnophiles par le biais d'ouvrages divers sur le vin: ce sont les lexiques cachés, listes de mots suivis d'une définition, réunis sous le titre de «Vocabulaire» ou Glossaire» et placés en général à la fin de l'ouvrage 3 .

L'ouvrage Le Goût du vin, publié en 1980 par le professeur et onologue Émile Peynaud, qui s'adresse aussi bien aux professionnels qu'aux œnophiles, fait date et référence : entièrement consacré à l'art de la dégustation, il en présente une étude scientifique et sociologique, ainsi qu'une réflexion sur le langage des dégustateurs «aux prises avec l'impuissance des mots», insistant sur l'importance que «les dégustateurs aient le même langage, qu'ils se comprennent et qu'ils se fassent comprendre, qu'ils emploient donc les mêmes mots pour les mêmes perceptions» (p. 152). Lui-même cite plus d'un millier de mots utilisés pour décrire les aspects visuel, olfactif et gustatif du vin. Soulignant la complexité et la variété de ce vocabulaire, il présente les essais de figuration rationnelle que les œnologues ont mis au point, figuration basée sur la notion d'équilibre entre les trois composants du vin: l'alcool, l'acidité et l'astringence. Cette représentation sous forme de schéma (notamment la définition graphique triangulaire de Vedel) permet de situer les qualificatifs les uns par rapport aux autres selon une échelle de qualité et d'intensité.

Peynaud distingue plusieurs «niveaux de terminologie» (p. 153) selon que le commentateur est un expert - anologue ou dégustateur de métier -, un amateur éclairé, ou un chroniqueur vinicole. Cette distinction induit des «niveaux de technicité» (Quemada 1978: 1150) des discours respectifs qui vont du plus précis - recherche du vocable adéquat, utilisation de termes codifiés -, au plus flou - manquant de rigueur, procédant par allusions et analogies, lyrique.

À titre de références pour cette étude, nous avons retenu 5 glossaires et dictionnaires consacrés à la dégustation ou, de façon plus générale, au domaine du vin, proposant des définitions: le «Glossaire de l'amateur de vin» figurant à la fin du Livre de l'amateur de vin de Norbert Got (GOT) (1967); le «Vocabulaire» de Vedel et al. (VEDEL) (cité plus haut) (1972); Les Mots du vin et de l'ivresse de Martine Châtelain-Courtois (COURTOIS) (1984); le «Glossaire» de l'ouvrage Connaître le vin de Jacques Puisais ${ }^{4}$ (PUISAIS) (1986); le Dictionnaire du vin d'Yves Renouil (RENOUIL) (1988). Les unités figurant à la nomenclature de ces répertoires, définis par les spécialistes du domaine, ont le statut de terme, même si certains sont considérés comme «imprécis» par leur auteur (VEDEL).

Les dictionnaires de langue n'ont pas été pris en compte puisqu'ils ne recensent en principe que les termes entrés dans l'usage général. Cependant, la consultation du Trésor de la Langue Française (TLF) a permis de constater la très faible représentativité des mots relatifs à la dégustation. Cette remarque en rejoint d'autres énoncées à propos de domaines connexes (le vocabulaire de l'ampélographie, voir Rézeau 1992). 


\section{Les comptes rendus de dégustation}

Les comptes rendus de dégustation d'où sont tirés les relevés étudiés ici sont rédigés par des journalistes-dégustateurs - ou dégustateurs-journalistes, dont la réputation dans le monde du vin n'est plus à faire et qui collaborent à des journaux spécialisés dont l'un, La Revue du vin de France, mensuel, vendu en kiosque, est le plus ancien et le plus largement diffusé et l'autre, Le Rouge et le blanc, trimestriel, est vendu sur abonnement, à diffusion plus restreinte. Ils s'adressent aux professionnels du vin et aux «nouveaux dégustateurs» (Albert 1989), public d'amateurs éclairés, membres de clubs de dégustation et consommateurs de littérature vinique.

Il est certain que le discours de la dégustation relève d'abord du code oral. On n'insistera pas sur les difficultés inhérentes aux études d'un corpus oral. Les comptes rendus constituent un corpus relativement homogène par rapport aux commentaires éparpillés dans des articles ou ouvrages divers et présentent au moins l'avantage de fixer par écrit des impressions et d'aider à leur mémorisation. En outre, leur présentation typographique (utilisation des italiques, de guillemets, etc.), indique un souci de fidélité et d'authenticité dans la transcription. Enfin, et ce n'est pas le moins important, ces textes constituent les références langagières pour le public qui les lit, c'est-à-dire les amateurs qui pratiquent la dégustation et viennent y chercher les informations souhaitées.

\section{Métaphore et vocabulaire spécialisé}

La métaphore et la métonymie sont traditionnellement considérées comme étant essentiellement des figures de styles relevant du discours poétique et c'est la raison pour laquelle les nombreux travaux qui s'y rapportent visent avant tout le discours littéraire ou poétique. Les diverses approches théoriques de ces tropes, qui ont été passées en revue et analysées dans plusieurs études (Molino 1979; Pavel 1991; Horne 1992), en soulignent la complexité. Notre propos n'est pas de réviser ces approches, mais d'envisager la métaphore d'un point de vue lexicologique, dans son rôle de «suppléance dans la dénomination en l'absence de terme propre» (Le Guern 1973: 68), à l'intérieur d'un domaine d'expérience, la dégustation du vin, dont l'univers référentiel est bien particulier.

Reposant sur la notion d'analogie, le processus métaphorique consiste dans le transfert d'un ou plusieurs sèmes d'un signe sur un autre signe (par ex. flamme pour amour), ou dans l'attibution à un signe existant d'une signification nouvelle pour désigner une réalité qui n'avait pas encore de terme propre (ex. créneau (d'une voiture)), processus qui s'opère en fonction de rapports de similitude ou de parenté plus ou moins étroits entre les réalités auxquelles les signes renvoient ou entre les représentations mentales que les locuteurs en ont.

Contrairement à la métonymie qui, impliquant une relation entre les réalités extralinguistiques, repose sur un rapport d'association, de contiguité, et opère sur un déplacement de référence (par ex. verre pour le contenu du verre, ou nez pour les perceptions olfactives), la métaphore opère sur un transfert sémique, c'est-à-dire qu'elle implique nécessairement l'existence d'au moins un sème commun entre les sémèmes. Cependant, la distinction entre emplois métaphoriques et emplois métonymiques n'est pas toujours facile à établir. Analysant la nature de la relation entre les sèmes communs dans les métaphores lexicalisées, Tournier (1985: 225-226) identifie plusieurs types de transfert: le «transfert d'identité» (rare), fondé sur une quasi-identité sémique; le «transfert par analogie» (le plus fréquent), fondé sur un sème perçu comme commun; le «transfert par association», dans lequel la relation entre les sèmes communs relèvent plus de l'association que de l'analogie et qui, par conséquent, se rapproche du processus métonymique. Quoi qu'il en soit, c'est bien la notion de transfert qui est sous-jacente aux réalisations métaphoriques et métonymiques. 
La métaphore a toujours été une ressource privilégiée pour la formation des vocabulaires spécialisés, particulièrement les vocabulaires des métiers et des techniques. Pavel (1991: 41) rappelle que le sens figuré ou tropologique d'un mot est vu traditionnellement comme «prêté à ce mot soit par le besoin de combler une lacune, soit par le désir d'obtenir un effet esthétique». Kocourek (1991: 167-168) distingue les «métaphores vives», ou tropes libres, non lexicalisés, qui procèdent de la comparaison figurée, impliquant «un élément de subjectivité, de complicité, de secret», et les «tropes lexicaux» ou tropes nécessaires, correspondant à une nouvelle acception faisant partie du système lexical et pour lesquels la fonction référentielle ne peut être mise en doute.

Quemada (1978: 1166-1169) met l'accent sur l'importance de l'identité des expériences des groupes professionnels qui permet la compréhension immédiate d'une métaphore spontanée (cohérence de l'univers référentiel) et favorise la «connivence imaginative» : celle-ci est à la base de créations métaphoriques à forte charge affective et expressive, qui relèvent donc de la fonction poétique, mais s'imposent et se lexicalisent d'autant mieux que le groupe est stable dans ses relations et ses expériences. Ces considérations énoncées à propos des vocabulaires techniques décrivant des réalités concrètes, s'appliquent aussi bien au domaine que nous étudions ici : l'activité de la dégustation est par définition une activité de groupe restreint, au sein duquel les participants se créent des références communes aboutissant, dans le meilleur des cas, à un consensus linguistique. Il ne s'agit pas que les membres du groupe aient une identité de goût: les préférences comme les seuils de perception divergent selon les individus. Mais il leur devient possible de les exprimer et d'être compris, ou, pour user d'une métaphore familière, d'«être sur la même longueur d'ondes».

\section{LES CHAMPS THÉMATIQUES À LORIGINE DES MÉTAPHORES}

Plusieurs champs thématiques sont à la source des bases métaphoriques dans le vocabulaire des dégustateurs d'aujourd'hui. En voici les principaux, regroupés dans des catégories volontairement larges (et pas toujours étanches):

- l'être humain (physique) : athlétique, chair, charnu, musclé...;

- l'être humain (mental) : aimable, expansif, réservé, sincère...;

- l'être humain (social): aristocratique, noble, racé...;

- la réalité spatiale (forme, volume, dimension) : ample, cônique, rond...;

- la réalité physique (propriétés physiques, état de la matière): dense, solide, souple...;

- le contact, le mouvement, le déplacement : attaque, fuyant, retour, suite, tapisser...;

- le temps (âge, durée, évolution) : avenir, court, longueur en bouche, sénile...;

- les sens (métaphores intersensorielles ou synesthésiques): asséchant, dur, ferme; frais, lisse...;

- le textile: vin en dentelles, étoffe, texture, trame...;

- la construction: architecture, charpente, bien/mal construit...;

Nous nous intéresserons à deux champs, celui de l'être humain physique et celui des réalités spatiales, qui sont bien représentés dans les relevés de notre corpus.

Rappelons que nous considérons comme lexicalisées les unités recensées et définies dans au moins l'un des cinq répertoires de référence. Les unités qui ne figurent pas en entrée dans ces répertoires sont précédées d'un ${ }^{\circ}$ (ex.: ${ }^{\circ}$ carrure).

Pour mieux rendre compte de l'organisation de ces unités à l'intérieur du vocabulaire de la dégustation, nous les présentons dans des regroupements renvoyant aux divers constituants et aspects du vin qu'elles décrivent, selon la pratique des dégustateurs, c'està-dire : 
la teneur plus ou moins élevée ou marquée, en:

- extrait sec (substance ou matière du vin) (sensations de consistance);

- acidité (sensations de vivacité);

- alcool (sensations d'ordre thermique);

- annins (sensations d'astringence, qui provoquent la constriction des muqueuses buccales);

- sucres et glycérol (sensations de gras, de moelleux, et, pour certains vins, saveur sucrée);

- l'intensité et de la persistance aromatiques (olfactive et gustative);

- l'évolution et la modification des qualités gustatives;

- la constitution générale et l'équilibre (rapport entre les divers éléments constitutifs; sensations et appréciations globales).

\section{L'être humain}

On sait que les métaphores anthropomorphiques constituent la source la plus riche dans la constitution de nombreux vocabulaires techniques concrets. Bien que non concret, le vocabulaire des impressions gustatives dans la dégustation du vin n'échappe pas à cette règle et cela d'autant plus que le vin possède une forte charge symbolique et qu'il est un produit organique «vivant» (Châtelain-Courtois 1984: 15).

\section{Les métaphores du corps humain}

extrait sec: amaigri, amaigrissement (subir un -), anémique, chair, chair (avoir de la -), chair (bien en -), charnu, chétif, décharné, dégingandé, 'efflanqué, élancé, 'enrobé, 'enveloppé, étriqué, fluet, 'orêle, gringalet, maigre, maigrelet, ' $m e n u$, mince, rabougri, squelettique, svelte;

acidité : nerf (avoir du -), nerveux, nervosité;

alcool : athlétique, ${ }^{\circ}$ carrure, ${ }^{\circ}$ corpulence, corpulent, enveloppé, 'épaulé, étriqué, ${ }^{\circ}$ imposant; alcool + tannins: ${ }^{\circ}$ muscle (avoir du $u$ ), musclé, ${ }^{\circ}$ musculature, vigoureux, ${ }^{\circ}$ vigueur;

constitution et équilibre : allure (avoir de l'-), ${ }^{\circ}$ colonne vertébrale, constitué (bien/mal -), constitution, corps (avoir du -), élégance, élégant, féminin, masculin, 'ossature, robuste, ${ }^{\circ}$ robustesse, ${ }^{\circ}$ squelette, viril.

Cette liste, comme celle qui suivra, est composée en grande partie d'adjectifs, de substantifs en moins grand nombre, de quelques verbes et de locutions verbales. Dans les commentaires de dégustation, la plupart des adjectifs sont susceptibles d'être modifiés par un adverbe à valeur intensive, ce qui a pour effet d'augmenter encore la variété de l'expression: légèrement, peu, faiblement, moyennement, assez, très, etc. De même, les substantifs apparaissent dans des collocations adjectivales, adverbiales ou nominales qui apportent une évaluation d'intensité dans l'impression ou le plaisir ressentis (valeur hédoniste): faible ou grande ${ }^{\circ}$ carrure, ${ }^{\circ}$ corpulence moyenne, manque de ${ }^{\circ}$ colonne vertébrale, ${ }^{\circ}$ musculature puissante, etc.

L'analogie avec le corps humain pourvu de chair est basée sur l'impression produite par le vin dans la bouche, impression de consistance et de plénitude due à la présence d'extrait sec et de glycérine. Le terme chair, lexicalisé, consigné dans tous les répertoires spécialisés consultés mais curieusement absent des dictionnaires de langue ${ }^{5}$, joue le rôle de terme-pôle, à partir duquel s'organisent les unités qui en décrivent la présence plus ou moins marquée de cet ensemble de constituants.

Le statut de terme de chair favorise la terminologisation de ses corrélats morphologiques charnu et décharné («qui a perdu la plus grande partie de sa chair»), ainsi que celle des antonymes de charnu: maigre et mince, qui sont des quasi-synonymes, maigre 
étant plus péjoratif que mince. L'expression du manque de chair se traduit à son tour par des corrélats morphologiques de maigre (amaigri, subir un amaigrissement, relatifs, comme décharné, à la perte des qualités de chair), et par une multiplicité de quasisynonymes allant de menu à squelettique, sortes de variantes lexicales à forte charge expressive, qui peuvent paraître fantaisistes mais qui sont sans opacité grâce aux relations synonymiques qu'elles entretiennent avec maigre/mince. Si les termes maigre et décharné étaient les seuls disponibles, ils seraient par là-même très réducteurs par rapport à la réalité à traduire. Le trait sémantique commun est en effet le manque de chair, les traits sémantiques périphériques des quasi-synonymes marquent la gradation et la péjoration : squelettique, qui indique un excès de maigreur avec une valeur nettement péjorative, exclut toute autre qualité du vin, alors que svelte indique que le vin n'est pas caractérisé par sa chair, mais n'exclut pas des qualités de vigueur ou de nervosité apportées par une teneur correcte en acidité, en alcool ou en tannins. Tout se passe comme si la relation perçue entre les signifiés en langue induisait, chez le dégustateur, dès lors qu'il cherche à préciser et moduler la complexité de ses impressions, l'emploi d'une échelle sémantiquement cohérente des signifiants.

Dans la description de la chair, les relations antonymiques à l'intérieur du champ métaphorique du corps humain sont peu nombreuses: avoir de la chair, bien en chair, charnu et bien nourri, sont les seuls qualificatifs opposés à la maigreur. En revanche, ils sont bien lexicalisés et d'un emploi fréquent dans le discours des dégustateurs. Les adjectifs 'enrobé et enveloppé, posent problème: selon les auteurs, enveloppé renvoie soit à l'aspect charnu (COURTOIS), soit à l'aspect vineux (présence marquée d'alcool) (VEDEL, RENOUIL). Dans nos attestations, ces deux unités sont associées à ample, puissant, gras, et à profondeur, concentration, qualités que ne saurait posséder un vin manquant de chair ni d'alcool. Nous nous trouvons ici devant un cas exemplaire de flou linguistique et de manque d'harmonisation terminologique, critiqués par les auteurs eux-mêmes. Leur usage courant comme quasi-synonymes de bien en chair appliqué à une personne, et d'antonymes de maigre/mince permettent leur intégration dans la série correspondante. C'est vraisemblablement cet aspect que COURTOIS a privilégié dans sa définition, laquelle s'appuie sur des usages attestés dans le type de discours que nous analysons ici, tandis que les définitions de VEDEL et RENOUIL sont celles d'œnologues-terminologues.

L'appréciation de l'acidité est faiblement représentée en nombre d'unités, mais celles-ci sont également cohérentes puisqu'elles gravitent morphologiquement autour de nerf, dont le sème de «vivacité» renvoie à l'impression ressentie. Cependant, la nervosité d'un vin n'exclut pas la présence d'autres constituants comme la chair ou les tannins. Elle est souvent associée à l'impression de vigueur, désignant par là une bonne combinaison d'alcool et d'acidité.

La présence d'alcool apporte force et chaleur au vin. Les unités métaphoriques qui décrivent la prédominance, ou, au contraire, la présence peu marquée de ce constituant, comportent le sème de «force physique», à travers l'image d'un corps humain «sportif»: athlétique, épaulé. Les sèmes de corpulent et imposant - grosseur et taille — assimilent ces adjectifs et les substantifs correspondants à l'idée de force. La combinaison alcool + tannins ajoute à la force une impression de tonicité traduite par vigueur, muscle et musclé, même sans présence marquée de chair. La trop faible teneur en alcool est le plus souvent traduite par des collocations nominales ou adjectivales construites à partir de ces unités: peu corpulent, faible ${ }^{\circ}$ carrure, manquant de / faible ${ }^{\circ}$ corpulence, manque de vigueur, etc. Le seul qualificatif à valeur négative est étriqué, antonyme d'épaulé.

Le regroupement constitution et équilibre fournit un ensemble apparemment plus hétérogène. Il s'articule autour de substantifs relatifs au corps humain dans sa représentation anatomique et physiologique, qui figurent la structure générale du vin: ${ }^{\circ}$ colonne 
vertébrale, constitution, ${ }^{\circ}$ ossature ${ }^{\circ}$ squelette. L'appréciation du rapport entre les éléments constitutifs se traduit par des collocations nominales ou adjectivales dont ces substantifs forment la base : faible ou bonne constitution, ${ }^{\circ}$ ossature solide, etc.

La locution corps (avoir $d u$-) a été classée dans cette série bien que certains auteurs la définissent plus particulièrement par rapport à la richesse en alcool. Il semble que 1'usage maintienne une certaine ambiguité, dans la mesure où la locution est employée tantôt dans ce sens, tantôt dans le sens donné par PUISAIS: «Terme qui allie les qualités d'un vin à la fois charnu, charpenté et chaud.» Dans cette acception, la locution est rejointe par robuste, dont les sèmes se rapprochent de «solide constitution». Le qualificatif viril et son synonyme récent ${ }^{\circ}$ masculin auraient pu être classés dans le regroupement alcool + tannins, puisqu'ils décrivent des vins possédant cette prédominance, mais, comme féminin, auquel ils s'opposent, ils correspondent à une appréciation générale de la constitution et sont définis par les auteurs par la notion d'équilibre modulée dans le sens de la vigueur pour les deux premiers, de la finesse et de la souplesse pour le troisième.

Les autres unités figurant dans cette série se rattachent à des appréciations plus subjectives, à caractère hédoniste. Plus floues, plus imprécises, elles renvoient à une image globale de l'apparence physique, le plus souvent positive: la notion d'élégance (équilibre dans la finesse) entraîne l'utilisation de la locution allure (avoir de l'-).

Dans ce champ thématique afférent au corps humain, on notera l'absence de quelques expressions connues cependant du grand public en raison de leur effet évocateur, comme avoir du corsage, avoir de la jambe, avoir de la cuisse: bien que signalées dans divers ouvrages (toujours entre guillemets), elles ne sont pas attestées dans notre corpus. Ce type de métaphores érotico-corporelles appartiennent au code oral et, même si elles sont encore répandues chez certains fréquenteurs de caves, il semble que les dégustateurs ne les jugent pas dignes de paraître dans leurs comptes rendus. On peut y voir un souci d'éviter un vocabulaire jugé trop imprécis peut-être, mais certainement aussi un souci de se démarquer précisément d'un public plus amateur que dégustateur, ainsi qu'une tendance à l'intellectualisation et à la sophistication du discours dégustatif.

Les unités analysées ici forment un ensemble relativement cohérent. Malgré un flou linguistique constaté pour un petit nombre d'entre elles et une tendance à la polysémie pour d'autres, les relations analogiques de type synonymique ou antonymique qui les unissent tendent vers une cohésion sémantique. Les unités non définies dans les répertoires viennent enrichir les séries déjà constituées en renforçant leur homogénéité morphologiquement et/ou sémantiquement.

\section{La réalité spatiale : forme, volume, dimension}

Tous les spécialistes de la dégustation constatent que les perceptions produites par le vin gardé et tourné dans la bouche pendant quelques instants sont assimilables à des impressions de forme, de volume et de consistance, la forme idéale étant la sphère, qui représente l'équilibre parfait. Peynaud (1983:157) a baptisé ce phénomène stéréodégustation. Dans son effort de description, le dégustateur va puiser, dans le vocabulaire relatif à la réalité spatiale, un certain nombre de vocables correspondant par analogie aux sensations reçues.

\section{Les métaphores de la réalité spatiale}

extrait sec: ample, ampleur, creux, épais, étalé, filiforme, large, longiligne, vide; alcool : abrupt, carré, massif; acidité : acéré, aigu, anguleux, pointu, relief (sans -); tannins : arêtes, aspérités ; intensité et persistance aromatique : ${ }^{\circ}$ ônique, ${ }^{\circ}$ cylindrique, long, longiligne ; 
constitution et équilibre: ${ }^{\circ}$ arrondi, carré, droit, forme, ${ }^{\circ}$ ligne, ${ }^{\circ}$ monolithique, plat, plein, plénitude, rectiligne, relief, rond, rondeur, 'silhouette, sphérique, volume, volumineux:

Selon sa proportion en extrait sec, un vin donne l'impression d'emplir plus ou moins bien la bouche; l'analogie est donc basée sur une forme et un volume attribués au liquide: ample, étalé, large quand la teneur en extrait sec (toujours alliée aux autres constituants comme l'alcool) est marquée, épais si elle est excessive, creux, filiforme, longiligne ou même vide (quasi-synonyme de maigre) si elle est insuffisante. Le qualificatif étalé est polysémique : défini par les auteurs dans le sens de «mince», c'est-à-dire «manquant de chair», il est également synonyme de ample dans nos relevés. De même, large, défini par RENOUIL par rapport à la force et à la vigueur, c'est-à-dire à la richesse en alcool, est utilisé comme quasi-synonyme de ample dans les commentaires de dégustations (connotation hédoniste). Il y a donc d'un côté, une métaphorisation opérée sur un emploi déjà figuré de large («large, donc fort»), intégrant ce vocable dans la série anthropomorhique avec athlétique, corpulent, (forte) ${ }^{\circ}$ carrure..., et, de l'autre, une re-figuration du sème spatial qui place le vocable dans la série ample..., comme si les dégustateurs rétablissaient spontanément une cohérence analogique selon leurs références.

L'expression d'une trop grande prédominance d'alcool est traduite par deux unités adjectivales dont les sèmes se rattachent à la notion de force et de violence : le contraire de la douceur (abrupt) et la lourdeur (massif).

Les impressions dues à l'acidité (saveur piquante, mordante) sont bien représentées: l'excès se manifeste dans le terme pointu, lexicalisé dans le vocabulaire du goût par analogie avec la sensation produite par une piqûre, et ses quasi-synonymes, acéré et aigu, dont se rapproche anguleux. Un manque d'acidité enlève son relief au vin, d'où plat, relief (sans -).

La présence dominante des tannins produit également une impression de relief agressif (âpreté et astringence sur les muqueuses buccales), mais comparable à une surface rugueuse : arêtes, aspérités.

L'intensité et la persistance aromatiques, non représentées dans les métaphores du corps humain, sont notées ici par des adjectifs plus proches du vocabulaire de la géométrie, qui rendent compte à la fois de la durée de la perception aromatique et de son volume: 'cônique (intensité qui diminue avec la durée), 'cylindrique (intensité égale pendant toute la durée), 'longiligne (intensité moyenne mais d'une bonne durée). Ces trois unités, absentes des répertoires, sont affectionnées de certains dégustateurs particulièrement sensibles (tous ne le sont pas) aux sensations de forme et de volume. L'emploi de vocables dont le contenu scientifique (en géométrie) se fait encore sentir bien qu'ils soient banalisés, répond à un besoin de précision en même temps qu'à une certaine sophistication.

On a vu que l'équilibre est la notion clé de la dégustation et que la forme sphérique en est le schéma idéal. Les termes forme, ligne, relief, silhouette, volume représentent les impressions d'ensemble propres à caractériser la constitution et l'équilibre. Les deux substantifs 'ligne et ${ }^{\circ}$ silhouette qui ne figurent dans aucun répertoire, sont des quasisynonymes de forme, défini par les professionnels mais relativement peu fréquent dans nos comptes rendus. Dans le discours de la dégustation, il semble qu'ils se prêtent plus facilement que forme à la détermination par un adjectif, par ex.: belle ligne, oligne structurée, 'silhouette fine / élégante.... Le succès de ligne peut également être recherché dans ses connotations positives actuelles (ligne/allure, ligne/sveltesse, ligne/esthétique). Ajoutons que les sèmes de silhouette justifieraient le classement du terme dans la série anthropomorphique.

L'équilibre harmonieux entre les éléments constitutifs se traduit par l'impression de rondeur dans les adjectifs rond et son quasi-synonymé sphérique, et son corrélat 
morphologique arrondi. À la notion de volume se rattachent plein et volumineux, indiquant la présence équilibrée et affirmée de tous les composants, ainsi que ${ }^{\circ}$ monolithique, qui exclue la rondeur. L'opposé de rond est plat, dont on peut dire qu'ils sont des quasiantonymes dans le vocabulaire de la dégustation, plat décrivant un vin sans saveur, marqué par la carence au niveau de chacun des composants. Aussi bien plat se situe-t-il à l'extrémité de l'échelle d'intensité par rapport aux termes exprimant l'excès d'un des éléments: massif, aigu/pointu, et peut fonctionner comme quasi-synonyme de étalé au sens de «manquant de chair».

Les adjectifs carré, droit, rectiligne sont appliqués aux impressions gustatives à partir d'un sème commun figuré «franc, net, honnête», qui renvoie à la notion d'absence de défaut. Nos attestations dans les commentaires de dégustation les signalent en relation syntagmatique avec d'autres qualificatifs comme strict, vigoureux, ce qui réactive une métaphorisation à partir du sème non figuré et renvoie au manque de rondeur. Là aussi, on trouve une opposition avec rond, mais sans que la qualité de la constitution ne soit mise en cause.

Les métaphores de la réalité spatiale sont riches et variées dans le vocabulaire de la dégustation. Les rapports analogiques référentiels sont assez cohérents, mais les relations analogiques entre les termes sont moins évidentes que dans le champ du corps humain.

\section{Disponibilité des métaphores}

Après avoir identifié et analysé les unités métaphoriques issues de deux champs thématiques, il nous a paru intéressant de chercher à apprécier leur disponibilité métaphorique. Pour ce faire, nous avons consulté le $T L F$ et relevé les emplois signalés «par analogie», «figuré» et «par métaphore», en excluant tout emploi dans un domaine spécialisé, technique ou scientifique. Ils sont regroupés selon les rubriques indiquées par le TLF :

- domaine des sensations et des perceptions:

odeur : corpulent ;

perceptions auditives (son, bruit, voix) : acéré, aigu, anémique, aspérités, corpulent, creux, filiforme, ${ }^{\circ}$ menu, plat, plein, plénitude, relief (auditif), rond, ténu, vide, vigoureux, vigueur; perceptions visuelles (+ couleur) : aigu, anémique, aspérités, plat, vigueur ; toucher : aspérités ;

- domaine de l'expression (expression, style, à propos d'une œuvre littéraire):

abrupt, acéré, aigu, ample, ampleur, 'arrondi, charnu, décharné, forme, 'ligne, musclé, nourri, nerf, ossature, plein, plénitude, pointu, robustesse, rond, rondeur, squelette, svelte, vide, vigoureux, vigueur;

domaine des arts et de l'esthétique:

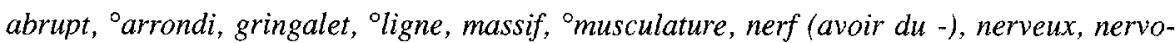
sité, ${ }^{\circ}$ ossature, rondeur, svelte, ${ }^{\circ}$ vigueur, volume ,

- emplois figurés non animé/abstrait:

amaigri, arêtes, ${ }^{\circ}$ carrure, ${ }^{\circ}$ colonne vertébrale, constitué (bien/mal -), ${ }^{\circ}$ muscle.

Ces résultats, même s'ils demandent à être affinés, mettent en évidence la propension d'un bon nombre de ces unités, issues de la langue commune, à la métaphorisation. Celles qui sont déjà inscrites dans les champs relatifs aux perceptions sensorielles à quelque degré que ce soit, auront d'autant plus de facilité à s'intégrer dans un autre champ sensoriel. Bien que les champs de l'expression et de l'esthétique ne soient pas liés d'un 
point de vue strictement référentiel au domaine que nous étudions, les unités qui y figurent renvoient à une appréciation, au regard porté par l'homme sur une production humaine soumise à une évaluation. Elles constituent un ensemble de vocables disponibles prêts à remplir un vide linguistique: «Lorsqu'un signifié 'en attente' ne peut s'exprimer que par une périphrase relativement longue, les conditions sont remplies pour que se produise cette captation de signifiant qu'est la métaphore.» (Tournier 1985 : 227).

\section{CONCLUSION}

Étudier les métaphores dans le vocabulaire de la dégustation peut paraître une gageure, étant donné la nature floue, imprécise du référent: les impressions gustatives. Les lacunes de notre langue en matière de vocabulaire spécifique de l'expression du goût, la difficulté de trouver les termes adéquats et le caractère hédoniste de la dégustation favorisent le recours au processus métaphorique qui génère une variété considérable de vocables souvent considérés comme fantaisistes et superflus. Cependant, une analyse détaillée révèle que le nombre de champs thématiques à l'origine des métaphores est restreint: moins de dix, parmi lesquels celui de l'être humain (physique et mental) et celui de la réalité spatiale, sont privilégiés.

Les unités s'organisent autour de notions-clés correspondant aux qualités gustatives principales, celles-ci étant liées à l'évaluation et à l'appréciation des éléments constitutifs du vin et à leur harmonie. Ces appréciations s'inscrivent dans des séries lexicales sur une échelle de qualité et d'intensité, à l'intérieur de laquelle les termes entretiennent des relations analogiques de type synonymique et antonymique, au sein d'un même champ ou entre deux champs: charnu, 'enrobé (corps humain)/ample, large (réalité spatiale); svelte, squelettique (corps humain)/creux, vide (réalité spatiale).

Le foisonnement lexical s'appuyant sur des relations analogiques entre les termes apparaît comme redondant, mais renforce en réalité la fonction cognitive en contribuant à cerner le référent.

L'apparition de vocables non encore recensés dans les répertoires spécialisés ('enrobé, ${ }^{\circ}$ menu, ${ }^{\circ}$ cônique ${ }^{\circ}$, monolithique) enrichit, sans la perturber, la cohésion des séries, morphologiquement et sémantiquement. Elle est d'autant plus facilitée qu'elle répond à la disponibilité métaphorique de ces vocables issus de la langue commune et déjà pourvus d'emplois métaphoriques dans des champs associés.

Il existe des cas d'ambiguité pour des termes définis dans ces mêmes répertoires, par rapport à l'usage des dégustateurs auteurs de comptes rendus. L'écart sémantique $s$ 'analyse en fonction du choix des sèmes retenus pour la production métaphorique. Cette confusion explique la polysémie de vocables comme étalé et large. Elle met en évidence le fait que l'absence de référent précis rend nécessaire la construction de références communes élaborées au sein d'un groupe autour d'une activité ou d'une pratique. De plus amples recherches sur le vocabulaire de la dégustation, et particulièrement sur les emplois métaphoriques, devraient pouvoir conduire à une clarification des termes, à leur définition et à une harmonisation linguistique indispensable à l'expression et à la communication dans le monde du vin.

\section{Notes}

1. Il est certain que des travaux philologiques et historiques apporteront un éclairage essentiel sur l'évolution des mots du goût lui-même (Henry 1986a; Bochnakowa 1988; Flandrin 1993b). Quelques dépouillements effectués dans des ouvrages scientifiques de la fin du XVIII et du début du XIX ${ }^{\mathrm{e}} \mathrm{s}$. comme ceux de Rozier et de Chaptal (voir $D D L$, vol. 43) mettent en lumière les lacunes des dictionnaires de langue à cet égard dans leur nomenclature comme dans leurs informations historiques.

2. Voir la liste bibliographique des Écrits sur le vin de 1600 à 1900 , dans l'ouvrage d'Émile Peynaud, Le Vin et les jours, Dunod, 1988, pp. 340-344. 
3. La liste de ces lexiques cachés serait trop longue à énumérer, vu l'abondance des publications viniques. Notons cependant que les journaux semi-spécialisés comme la Revue du vin de France ont proposé également dans les années 1970 des «Fiches de vocabulaire».

4. Jacques Puisais, fondateur de l'Institut Français du Goût à Tours.

5. La documentation des $D D L$ sous presse fournit une attestation de chair en 1851.

\section{RÉFÉRENCES}

ALBERT, Jean-Pierre (1989) : «La nouvelle culture du vin», Terrain 13, Boire, octobre, pp. 117-124.

BARTHES, Roland (1957): Mythologies, Paris, Seuil.

BOCHNAKOWA, Anna (1988) : «L'opposition sec : doux se rapportant au vin dans quelques langues romanes», Studia Romanica Posnaniensia, XIII, pp. 9-14.

CHÂTELAIN-COURTOIS, Martine (1984): Les Mots du vin et de l'ivresse, Paris, Belin.

COUTIER, Martine (sous presse) : «La langue du dégustateur: des mots et des vins», Communication, Colloque «Les mots pour dire le goût», Dole, 14 novembre 1992, Académie franc-comtoise du Goût.

COUTIER, Martine (sous presse): Datations et Documents lexicographiques, sous la dir. de B. Quemada, vol. 43, Matériaux pour l'histoire du vocabulaire français réunis par M. Coutier, CNRS, INaLF, Paris, Klincksieck (sous presse, 1994).

$D D L=$ Datations et documents lexicographiques (sous presse): sous la dir. de B. Quemada, Matériaux pour l'histoire du vocabulaire français, CNRS, INaLF, Klincksieck, 41 volumes parus, vol. 42 et 43 sous presse.

FAURION, Annick (1992) : «Mille et une saveurs et seulement quatre mots pour le dire», L'Amateur de Bordeaux, $\mathrm{n}^{\circ}$ hors série, Le Goût, juin, pp. 19-24.

FISCHLER, Claude (1992) : «Savoir savourer et savourer le savoir», L'Amateur de Bordeaux, $\mathrm{n}^{\circ}$ hors série, Le Goût, juin, pp. 39-41.

FLANDRIN, Jean-Louis (1993a) : «Le goût a son histoire», Autrement, série Mutations, Le mangeur, $\mathrm{n}^{\circ} 138$, juin, pp. 147-158.

FLANDRIN, Jean-Louis (1993b) : cité in L'Amateur de Bordeaux, n 41, décembre, p. 64.

GOT, Norbert (1967) : Le Livre de l'amateur de vins, Perpignan, chez l'auteur.

GUILLARD, Alexandre (1993) : «La dimension sociale de la dégustation», L'Amateur de Bordeaux, $\mathrm{n}^{\circ} 41$, décembre, pp. 62-64.

GUINARD, Jean-Xavier (1992) : «La dimension olfactive de la dégustation et les descripteurs du vin», L'Amateur de Bordeaux, $\mathrm{n}^{\circ}$ hors série, juin, pp. 28-33.

HENRY, Albert (1971): Métonymie et métaphore, Paris, Klincksieck.

HENRY, Albert (1986a) : «À propos d'un texte œnologique en ancien français», Bulletin de la classe des lettres et des sciences morales et politiques, $5^{\mathrm{e}}$ série, tome LXXII, 1-3, pp. 16-29.

HENRY, Albert (1986b) : «Un texte cenologique de Jofroi de Waterford et Servais Copale», Romania, tome 107, pp. 1-37.

HENRY, Albert (1991): «La Bataille des Vins», Bulletin de la classe des lettres et des sciences morales et politiques, $6^{\mathrm{e}}$ série, tome II, pp. 6-9, pp. 203-248.

HORNE, Christine (1992): «La métaphore : quelques définitions contemporaines», ALFA, vol. 5, pp. 179-180

HUET, Myriam (1993): «Les qualités du vin selon l'œnologue», L'Amateur de Bordeaux, $\mathrm{n}^{\circ}$ 40, septembre, pp. 76-79.

L'imaginaire du vin (1983): Actes du Colloque du 15-17 octobre 1981 publiés par Max Milner et Martine Châtelain, Marseille, Jeanne Laffitte.

KOCOUREK, Rostislav (1991): La langue française de la technique et de la science, $2^{\mathrm{e}}$ ed., Wiesbaden, Oscar Brandstetter Verlag (RFA), Diff. Documentation française.

LE GUERN, Michel (1973) : Sémantique de la métaphore et de la métonymie, Paris, Larousse.

LE MAGNEN, J. (1962): Vocabulaire technique des caractères organoleptiques et de la dégustation des produits alimentaires, Paris, Centre national de Coordination des Études et Recherches sur la Nutrition et I'Alimentation.

MARTIN, Éveline (1983): Reconnaissance de contextes thématiques dans un corpus textuel, CNRS, INaLF, Coll. «Études de sémantique lexicale», Paris, Didier Érudition.

MATORÉ, Georges (1962) : L'Espace humain, Paris, éd. du Vieux Colombier.

MCLEOD, Patrick (1987): «Si on ne sent pas pareil, c'est qu'on «nez» pas pareil», Entretien avec Patrick McLeod, Autrement, série Mutations, Odeurs, $\mathrm{n}^{\circ}$ 92, septembre, pp. 74-79.

MOLINO, Jean, SOUBLIN, Françoise et Joëlle TAMINE (1979) : «Présentation : problèmes de métaphore», Langages, 54, juin, 5-40.

NORMAND, Claudine (1976): Métaphore et concept, Paris, PUF.

PAVEL, Silvia (1991) : «Changement sémantique et terminologie», Meta, vol. 36, n 1, pp. 41-48.

PEYNAUD, Émile (1980) : Le Goût du vin, Paris, Dunod, Bordas.

PIJASSOU, René (1977) : «Naissance d'un vocabulaire», Raymond Dumay (dir.), Le Vin de Bordeaux et du Haut-Pays, Paris, éd. Montalba, pp. 169-173. 
PUISAIS, Jacques (1986) : Connaître le vin, Tours, NR éditions.

PUISAIS, Jacques (1989) : «Le goût, sens des sens», Autrement, série Mutations, nº 108, Nourritures, septembre, pp. 25-31.

QUEMADA, Bernard (1978) : «Technique et langage», Histoire des techniques, Paris, Gallimard, pp. 1146-1240. QUEMADA, Bernard : voir: $D D L$ et $T L F$.

RABOURDIN, Jean R. (1989) : Vocabulaire international de la dégustation, Elvire Éditions.

RENOUIL, Yves (dir.) (1988) : Dictionnaire du vin, avec la collab. de Claude Féret, nouv. éd., Boulognesur-Seine, Sézame.

RÉZEAU, Pierre (1992) : «Pour une étude historique et étymologique des noms de cépage en français», Cahiers de lexicologie, 60, 1992-1, pp. 115-129.

La Revue du vin de France, mensuel, Paris, fondé en 1927.

Le Rouge et le blanc, trimestriel, Paris, Les Éditions du vin, fondé en 1983.

ROVENTA-FRUMUSANI, Daniela (1989) : «Cognitif et expressif dans l'étude de la métaphore», Revue roumaine de linguistique, tome XXXIV, $\mathrm{n}^{\circ} 6, \mathrm{pp} .523-529$.

$T L F=$ Trésor de la langue française (1971-1993) : publié sous la direction de P. Imbs, puis de B. Quemada, CNRS, INaLF, Paris, Gallimard, 15 vol. parus.

TOURNIER, Jean (1985) : Introduction descriptive à la lexicogénétique de l'anglais contemporain, Paris, Champion.

VEDEL, André et Gaston CHARLE, Pierre CHARNAY, Jules TOURMEAU (1972) : Essai sur la dégustation des vins, INAO, Macon, S.E.I.V. 